Originalien

Z. Epileptol. 2019·32:326-338 https://doi.org/10.1007/s10309-019-00287-7 Eingegangen: 5. August 2019

Angenommen: 30. August 2019

Online publiziert: 30. September 2019

(c) Der/die Autor(en) 2019

Malin Kalski ${ }^{1,2} \cdot$ Susanne Schubert-Bast ${ }^{1,2,3} \cdot$ Matthias Kieslich $^{1,3} \cdot$ Anne-Christine Leyer ${ }^{1,2,3} \cdot$ Tilman Polster $^{4}$ - Arne Herting ${ }^{4}$ - Thomas Mayer ${ }^{5}$. Regina Trollmann ${ }^{6}$. Bernd A. Neubauer ${ }^{7}$. Ulrich Bettendorf ${ }^{8}$. Thomas Bast ${ }^{910}$. Adelheid Wiemer-Kruel ${ }^{9} \cdot$ Sarah von Spiczak ${ }^{11}$. Gerhard Kurlemann ${ }^{12,13}$. Markus Wolff $^{14,15}$. Gerhard Kluger ${ }^{16,17}$. Joe Carroll ${ }^{18}$. Daniel Macdonald ${ }^{18}$. Clive Pritchard ${ }^{18}$. John Irwin ${ }^{19} \cdot$ Karl Martin Klein ${ }^{1,2,20} \cdot$ Felix Rosenow $^{1,3}$. Adam Strzelczyk ${ }^{1,2,21} \cdot$ Lara Kay $^{1,2}$

'Epilepsiezentrum Frankfurt Rhein-Main und Zentrum für Neurologie und Neurochirurgie, GoetheUniversität Frankfurt, Frankfurt am Main, Deutschland; ${ }^{2}$ LOEWE Center for Personalized Translational Epilepsy Research (CePTER), Goethe-Universität Frankfurt, Frankfurt am Main, Deutschland; ${ }^{3}$ Klinik für Neuropädiatrie, Goethe-Universität Frankfurt, Frankfurt am Main, Deutschland; ${ }^{4}$ Epilepsiezentrum Bethel, Bielefeld, Deutschland; ${ }^{5}$ Epilepsiezentrum Kleinwachau, Dresden-Radeberg, Deutschland; ${ }^{6}$ Abteilung für Neuropädiatrie, Friedrich-Alexander Universität, Erlangen, Deutschland; ${ }^{7}$ Abteilung für Kinderneurologie, Sozialpädiatrie und Epileptologie, Justus-Liebig-Universität Gießen, Gießen,

Deutschland; ${ }^{8}$ Neuropädiatrische Praxis, Hirschaid, Deutschland; ${ }^{9}$ Epilepsiezentrum Kork, Kehl-Kork, Deutschland; ${ }^{10}$ Medizinische Fakultät, Universität Freiburg, Freiburg, Deutschland; " Norddeutsches Epilepsiezentrum für Kinder und Jugendliche, Schwentinental-Raisdorf, Deutschland; ${ }^{12}$ Abteilung für Neuropädiatrie, Universität Münster, Münster, Deutschland; ${ }^{13}$ Klinik für Kinder- und Jugendmedizin, Bonifatius-Hospital Lingen, Lingen (Ems), Deutschland; ${ }^{14}$ Abteilung für Neuropädiatrie, Universität Tübingen, Tübingen, Deutschland; ${ }^{15}$ Zentrum für Sozialpädiatrie und Neuropädiatrie, Vivantes Klinikum Neukölln, Berlin, Deutschland; ${ }^{16}$ Epilepsiezentrum Vogtareuth, Schön-Kliniken, Vogtareuth, Deutschland; ${ }^{17}$ Institut für Rehabilitation, Transition und Palliation von neurologisch kranken Kindern, PMU Salzburg, Salzburg, Österreich; ${ }^{18}$ Wickenstones Ltd., Goring Heath, Vereinigtes Königreich; ${ }^{19}$ Zogenix International Limited, Maidenhead, Vereinigtes Königreich; ${ }^{20}$ Department of Clinical Neurosciences, Cumming School of Medicine, University of Calgary, Calgary, Kanada; ${ }^{21}$ Epilepsiezentrum Hessen und Klinik für Neurologie, Philipps-Universität Marburg, Marburg (Lahn), Deutschland

\title{
Klinische Charakteristika, Ressourcenverbrauch, Lebensqualität und Versorgungssituation beim Dravet-Syndrom in Deutschland
}

auf $1 \mathrm{zu} 20.000$ Lebendgeburten und die Prävalenz auf 2 von 100.000 geschätzt [6-10]. Im Jahr 2017 wurde in Europa eine Prävalenz des DS zwischen 11.345 und 13.721 Personen angenommen [10].

Dem DS liegt in ca. $85 \%$ eine Mutation im SCN1A-Gen zugrunde [8], das für einen spannungsabhängigen Natriumkanal („sodium channel protein type 1 subunit alpha“) kodiert [11]. Die Mortalität und Morbidität des DS sind aufgrund von Unfällen, Status epilepticus (SE) und des Risikos des plötzlich auftretenden, ungeklärten Todes bei Epilepsie („sudden unexpected death in epilepsy patients" [SUDEP]) hoch $[5,12,13]$. Therapeutisch stehen v. a. Valproat, Brom, Clo- bazam und Topiramat als wirksame $\mathrm{Me}$ dikamente zur Verfügung [14], zudem ist Stiripentol (in Kombinationstherapie mit Clobazam und Valproat) speziell für das DS zugelassen [15]. Ketogene Diätformen gehören ebenfalls zu den therapeutischen Optionen [16]. Dennoch ist die Therapie des DS zum aktuellen Zeitpunkt eine Herausforderung, da kaum Patienten anfallsfrei werden. Mit Cannabidiol [17] und Fenfluramin [18-20] stehen weitere Medikamente zur Therapie des DS vor der Zulassung.

Insgesamt ist die Versorgung von $\mathrm{Pa}$ tienten mit therapierefraktärer Epilepsie, kognitiven Einschränkungen sowie weiteren Begleiterkrankungen mit weit- 


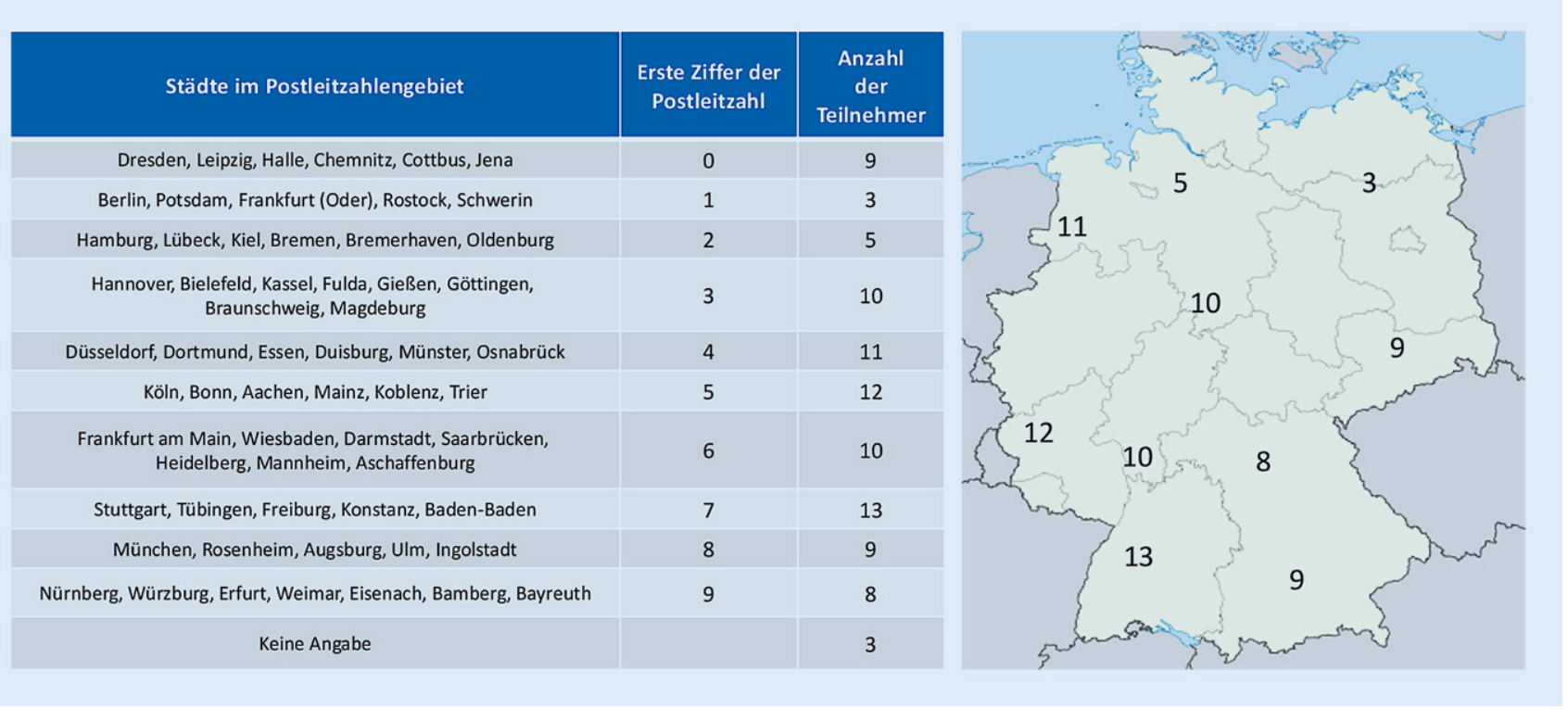

Abb. $1 \Delta$ Geografische Verteilung der Teilnehmer nach der ersten Ziffer der Postleitzahl (Deutschlandkarte entnommen aus https://commons.wikimedia.org/wiki/File:Germany_location_map.svg; Autor: NordNordWest [CC BY-SA 3.0 (https:// creativecommons.org/licenses/by-sa/3.0)])

reichenden sozioökonomischen Auswirkungen auf die Familie und das Gesundheitssystem verbunden. Ziel dieser Studie ist es, diese Auswirkungen im Detail zu erfassen und so auf die Krankheitslast und die damit verbundene Notwendigkeit der Verbesserung der Versorgung von Patienten mit einem DS hinzuweisen.

\section{Methoden}

Die Studie wurde als eine prospektive, multizentrische Querschnittsstudie konzipiert und schloss Patienten mit einem DS und deren Eltern bzw. Erziehungsberechtigte aus ganz Deutschland ein. Die Eltern wurden über Epilepsiezentren sowie Neuropädiater (Bielefeld, Dresden, Erlangen, Frankfurt am Main, Gießen, Hirschaid, Kiel, Kork, Münster, Tübingen, Vogtareuth) und über die Selbsthilfegruppe betroffener Eltern, dem Dravet-Syndrom e. V. (www.dravet. de, Markkleeberg, Deutschland), kontaktiert. Patienten, bei denen Zweifel an der Diagnose eines Dravet-Syndroms bestanden, wurden nicht in die Studie eingeschlossen.

Der Einschluss erfolgte nach Erhalt der schriftlichen Einverständniserklärung der Eltern bzw. Erziehungsberechtigten. Es wurde die aktuelle Klassifika- tion von epileptischen Anfällen, Epilepsiesyndromen und des Status epilepticus der International League Against Epilepsy (ILAE) zugrunde gelegt [21-23]. Für die Studie lag ein positives Votum der Ethikkommission der GoetheUniversität Frankfurt vor, zudem erfolgte der Eintrag der Studie in das Deutsche Register Klinischer Studien (www.drks.de) unter der Studiennummer DRKS00011894. Es wurden die Leitlinien für die Auswertung von Beobachtungsstudien (STROBE [Strengthening the Reporting of Observational Studies in Epidemiology]) befolgt [24].

Eltern bzw. Erziehungsberechtigte von Patienten mit DS wurden gebeten, einen retrospektiven Fragebogen zu den letzten 3 bzw. 12 Monaten und ein prospektives Tagebuch über den Verlauf von 3 Monaten auszufüllen. Der Fragebogen, der in früheren Studien [25-27] validiert und für die Anwendung bei Patienten mit DS angepasst wurde, umfasste 29 Fragen zu Krankheitsmerkmalen und zur Nutzung von Gesundheitsressourcen (direkte Kosten) sowie zu Einschränkungen der Arbeitstätigkeit der Eltern bzw. Erziehungsberechtigten (indirekte Kosten). Das prospektive Tagebuch sammelte Daten zu epileptischen Anfällen, Arztbesuchen, Therapien und Krankenhausaufenthalten sowie zu Medikamen- teneinnahmen und Zuzahlungen. Das Ziel des Tagebuches lag in der tagesgenauen Erfassung von Anfällen und der Inanspruchnahme von Ressourcen sowie der Validierung der Daten des retrospektiven Fragebogens.

Es wurden 3 Instrumente zur Erfassung der Lebensqualität (engl. Quality of Life [QoL]) in den Fragebogen aufgenommen. Die QoL-Werte der Kinder und Jugendlichen zwischen 4 und 17 Jahre wurden anhand der altersangepassten und gut etablierten KINDL-Fragebögen ermittelt, die by proxy durch die Eltern bzw. Erziehungsberechtigten ausgefüllt wurden [28]. Als Messgrößen für die QoL der Eltern bzw. der Erziehungsberechtigten wurden das Beck Depressions-Inventar II (BDI-II) [29] und die EuroQol Skala mit 5 Dimensionen und 3 Leveln (EQ-5D-3L) sowie die visuelle Analogskala (EQ-VAS) [30] verwendet.

Die Fragebögen und Tagebücher wurden in Papierform auf Deutsch ausgefüllt und konnten anonym per Post zurückgesendet werden. Die Daten der Fragebögen wurden zwischen April 2017 und Januar 2018 erhoben und die Tagebücher für 3 Monate nach dem Ausfüllen der Fragebögen geführt.

Die Methodik der Studie, Ergebnisse zu direkten und indirekten Kosten [31], und eine genaue Analyse der antikonvul- 
Z. Epileptol. 2019.32:326-338 https://doi.org/10.1007/s10309-019-00287-7

(c) Der/die Autor(en) 2019

M. Kalski $\cdot$ S. Schubert-Bast $\cdot$ M. Kieslich $\cdot$ A.-C. Leyer $\cdot$ T. Polster $\cdot$ A. Herting $\cdot$ T. Mayer $\cdot$ R. Trollmann $\cdot$ B. A. Neubauer $\cdot$ U. Bettendorf $\cdot$ T. Bast $\cdot$ A. Wiemer-Kruel · S. von Spiczak · G. Kurlemann · M. Wolff · G. Kluger · J. Carroll · D. Macdonald · C. Pritchard · J. Irwin · K. M. Klein · F. Rosenow · A. Strzelczyk • L. Kay

\section{Klinische Charakteristika, Ressourcenverbrauch, Lebensqualität und Versorgungssituation beim Dravet-Syndrom in Deutschland}

\section{Zusammenfassung}

Fragestellung. Ziel der prospektiven, multizentrischen Studie ist die Erfassung klinischer Charakteristika, des Ressourcenverbrauches sowie der Lebensqualität bei Patienten mit der Diagnose eines Dravet-Syndroms (DS) und deren Eltern in Deutschland.

Methoden. Die Datenerhebung erfolgte mit einem validierten retrospektiven Fragebogen über 3 bzw. 12 Monate sowie mit einem prospektiven Tagebuch über 3 Monate. Es wurden Daten zur Anfallssituation, Medikamenteneinnahme, Therapieinanspruchnahme sowie zu direkten und indirekten Kosten und zur Lebensqualität erhoben. Die Fragebögen und das Tagebuch wurden den Eltern über den Dravet-Syndrom e.V. sowie beteiligte Zentren ausgehändigt.

Ergebnisse. Der Fragebogen wurde von 93 Eltern der DS-Patienten und das Tagebuch von 77 ausgefüllt. Das mittlere Alter der Patienten betrug 10 Jahre (Spannweite
15 Monate bis 33,7 Jahre). Die Zeit bis zur Syndromdiagnose eines DS war in den letzten beiden Jahrzehnten deutlich kürzer. In den letzten 12 Monaten ereignete sich bei $95 \%$ der Patienten mindestens ein epileptischer Anfall. Als anfallsauslösende Faktoren wurden Fieber (93,4\%), Aufregung (56\%), Schlafmangel $(51,6 \%)$ und starke körperliche Anstrengung $(50,5 \%)$ berichtet. Die Lebenszeitprävalenz des Status epilepticus lag bei $77 \%$, und bei $28 \%$ war mindestens 1 Episode eines Status epilepticus innerhalb des letzten Jahres aufgetreten. Die Lebensqualität (QoL) der Patienten war niedriger als die der Allgemeinbevölkerung, und von $46 \%$ der Eltern wurden Depressionssymptome berichtet. Die direkten Kosten in 3 Monaten betrugen $6043 €$ pro Patient. Den größten Kostenfaktor stellten die stationären Kosten dar $(1702 €)$, gefolgt von den Leistungen für Pflege $(1130 €)$, den Kosten für die antikonvulsiven Medikamente (892 $€$ ) und für Therapien $(559 €)$. Bei den Müttern betrugen die gesamten indirekten Kosten $4399 €$ und bei den Vätern $391 €$ bezogen auf 3 Monate. Schlussfolgerung. Das Dravet-Syndrom ist mit häufigen, oft therapierefraktären epileptischen Anfällen und Status epilepticus vergesellschaftet. Diese Studie zeigt die erhebliche Krankheitslast und die damit verbundenen Einschränkungen in der Lebensqualität sowie die hohen direkten und indirekten Kosten auf. Um eine Verbesserung der Lebensqualität bei Patienten mit DS und deren Eltern zu erreichen, bedarf es neuer Therapie- und Versorgungskonzepte.

Schlüsselwörter

Epilepsie $\cdot$ Anfall $\cdot$ Antikonvulsiva $\cdot$ Status epilepticus · "Severe Myoclonic Epilepsy of Infants"

\section{Clinical characteristics, resource utilization, quality of life and care situation for patients with Dravet syndrome in Germany}

\section{Abstract}

Introduction and objective. Dravet syndrome (DS) is a rare epileptic and developmental encephalopathy associated with cognitive impairment and delayed development due to genetic mutations predominantly in the SCN1A gene. This multicenter, prospective survey-based study collected data about the clinical characteristics, health care resource utilization, costs and quality of life (QoL) of patients with DS and their caregivers in Germany.

Methods. A retrospective questionnaire covering the previous 3-12 months was used in addition to a prospective diary over 3 months to confirm the retrospective data and to obtain detailed information about indirect costs, QoL, medication, seizures and health care resource utilization. The questionnaires and the diary were handed out to the parents via Dravet-Syndrome e. V. and participating centers.
Results. The questionnaire and diary were completed by 93 and 77 caregivers of DS patients, respectively. Patient mean age was 10 years (range: 15 months -33.7 years), whereby the time to diagnosis was significantly lower in the last two decades. In the previous 12 months, $95 \%$ of patients experienced at least one epileptic seizure. Fever (93.4\%), excitement (56\%), sleep deprivation (51.6\%) and physical exertion $(50.5 \%)$ were reported as the factors that triggered seizures. The lifetime prevalence of status epilepticus was $77 \%$, with $28 \%$ having had at least one episode of status epilepticus within the last year. Patient QoL was lower compared with the general population, with $46 \%$ of the parents reporting symptoms of depression. The 3-month direct healthcare costs were $6043 €$ per patient, the largest components of which were inpatient costs $(1702 €)$ followed by nursing care services
(1130€), anticonvulsant drugs (892€) and ancillary treatment $(559 €)$. The 3-month total indirect cost for mothers was $4399 €$ and for fathers $391 €$.

Conclusion. The DS is associated with frequent and often treatment refractory seizures, including status epilepticus as well as various comorbidities. This study shows the substantial associated socioeconomic impact of the disease, with high direct and indirect costs and reduced QoL for patients and the caregivers. In order to achieve an improvement in the QoL for patients with DS and caregivers, new treatment and care concepts are necessary.

Keywords

Epilepsy · Seizure · Anticonvulsants · Status epilepticus - Severe Myoclonic Epilepsy of Infants 


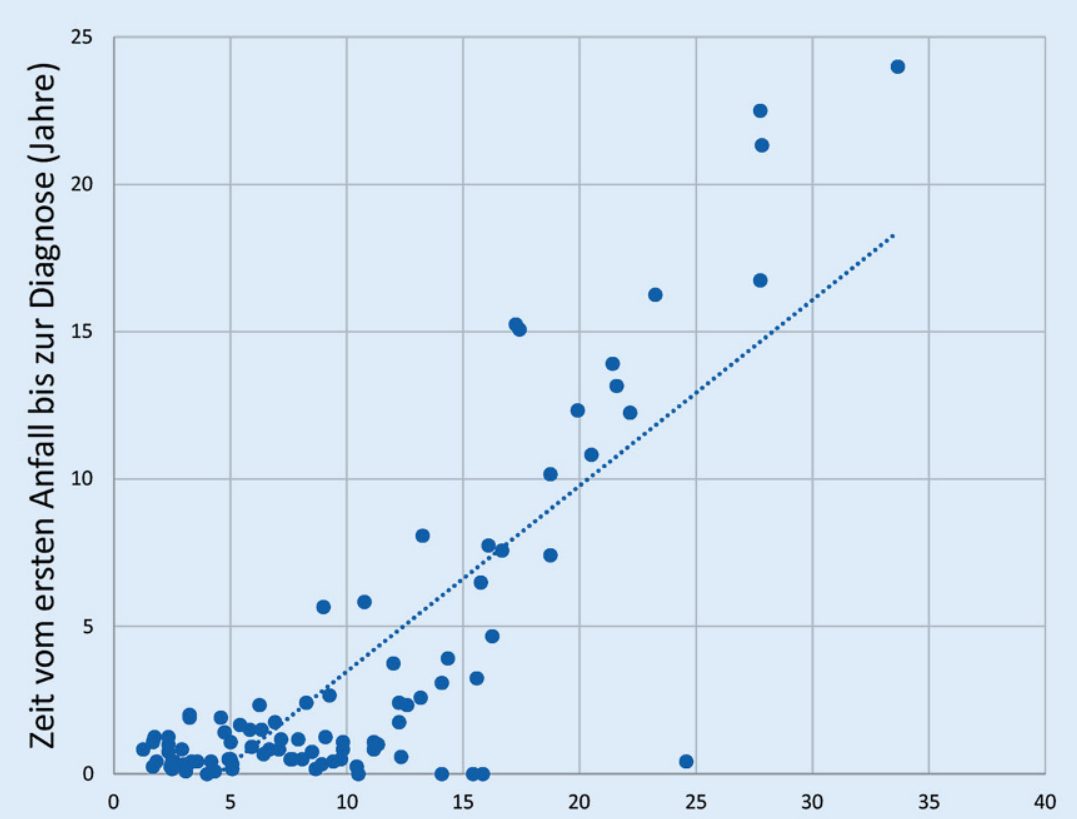

a

Alter bei Studieneinschluss (Jahre)

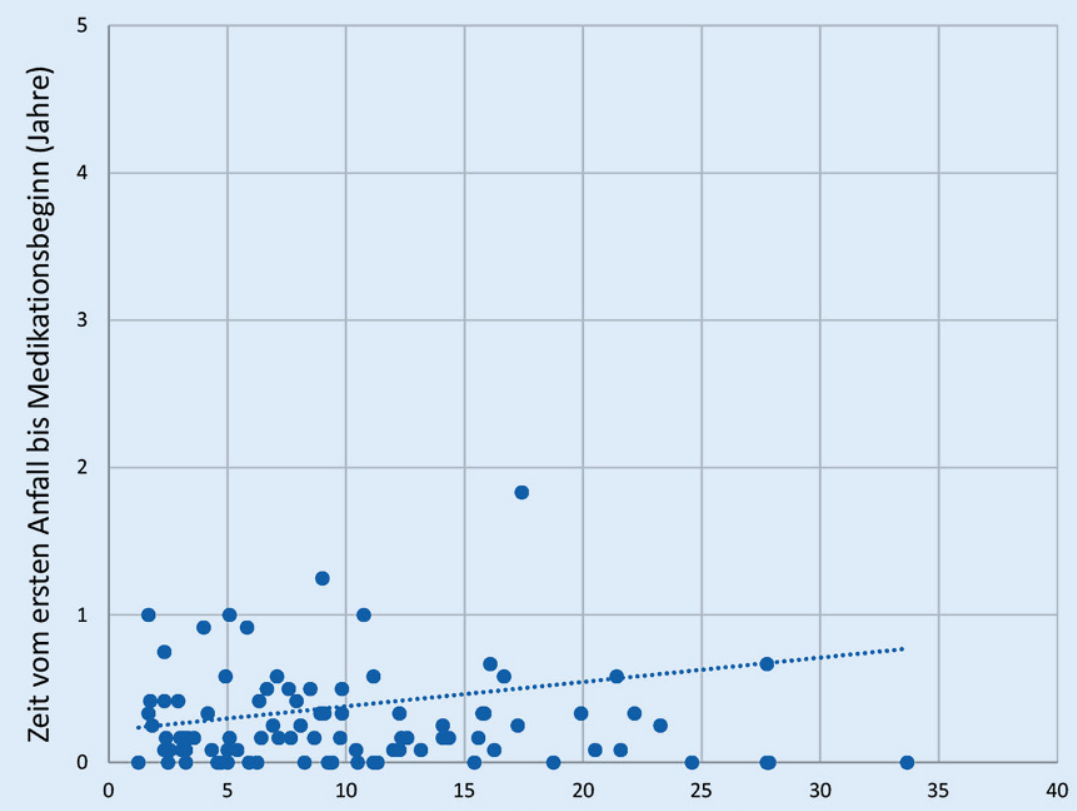

b

Alter bei Studieneinschluss (Jahre)

Abb. $2 \Delta$ a Altersabhängige Darstellung der Zeit vom ersten Anfall bis zur Diagnosestellung sowie b vom ersten Anfall bis zum Therapiebeginn mit Antikonvulsiva (ein Ausreißer mit einer Dauer von 122 Monaten bis zum Therapiebeginn ist nicht dargestellt)

siven Therapie [32], wurden bereits veröffentlicht. Verweise sind im Ergebnisteil des Artikels entsprechend gekennzeichnet.

\section{Statistik}

Die Daten wurden mit dem Statistikprogramm IBM SPSS Statistics 25 (IBM Corp., Armonk, NY, USA) analysiert. Metrische Variablen wurden als Mittelwert \pm Standardabweichung, Median, Minimum und Maximum angegeben, während kategorische Variablen als absolute Zahl und Prozentzahl dargestellt wurden.

\section{Ergebnisse}

\section{Demografische Daten und klinische Charakteristika}

Insgesamt nahmen 93 Eltern bzw. Erziehungsberechtigte von Patienten mit der Diagnose eines DS an der Studie teil, die den retrospektiven Fragebogen ausgefüllt zurückgeschickt haben. Das prospektive Tagebuch wurde von 75 dieser Studienteilnehmer $(80,6 \%)$ und 2 weiteren Probanden zurückgeschickt, die nicht in derStichprobe des Fragebogens enthalten waren. Das mittlere Patientenalter betrug 10 Jahre (Standardabweichung [SA] 7,1; Median 8,5; Spannweite [SW] 15 Monate bis 33,7 Jahre), wobei der Anteil der männlichen Patienten bei $53 \%$ lag (47\% weibliche Patienten). Das Durchschnittsalter der Mütter lag bei 42 Jahren (SA 7,6; SW 28 bis 62 Jahre) und das der Väter bei 45 Jahren (SA 7,7; SW 29 bis 70 Jahre) [31].

Die Rekrutierung der Studienteilnehmer gelang gleichmäßig über das Bundesgebiet und ist in $\bullet$ Abb. 1 anhand der ersten Ziffer der Postleitzahl visualisiert.

Das Durchschnittsalter bei Krankheitsbeginn betrug 5,9 Monate (SA 3,7; Median 5; SW 0 bis 26 Monate). Die mittlere Dauer zwischen dem ersten epileptischen Anfall und der Diagnosestellung belief sich auf 44,7 Monate (SA 66,5; SW 0 bis 288 Monate), wobei die Zeitdauer bis zur Diagnosestellung insbesondere bei den älteren Patienten (bei Studieneinschluss 12 Jahre und älter) länger war. Die • Abb. 2a zeigt die Dauer in Jahren vom Zeitpunkt des ersten Anfalles bis zur Diagnosestellung eines DS in Abhängigkeit von dem Alter bei Studieneinschluss und macht die längere Latenz bis zur Diagnosestellung bei den Patienten deutlich, die sich aktuell in Transition oder im Erwachsenenalter befinden. Die Dauer zwischen dem Zeitpunkt des ersten Anfalles und der Einleitung einer antikonvulsiven Therapie betrug im Durchschnitt 4,8 Monate (SA 13,1; SW 0 bis 122 Monate). Die - Abb. 2b zeigt die konsistent kurze Zeit 

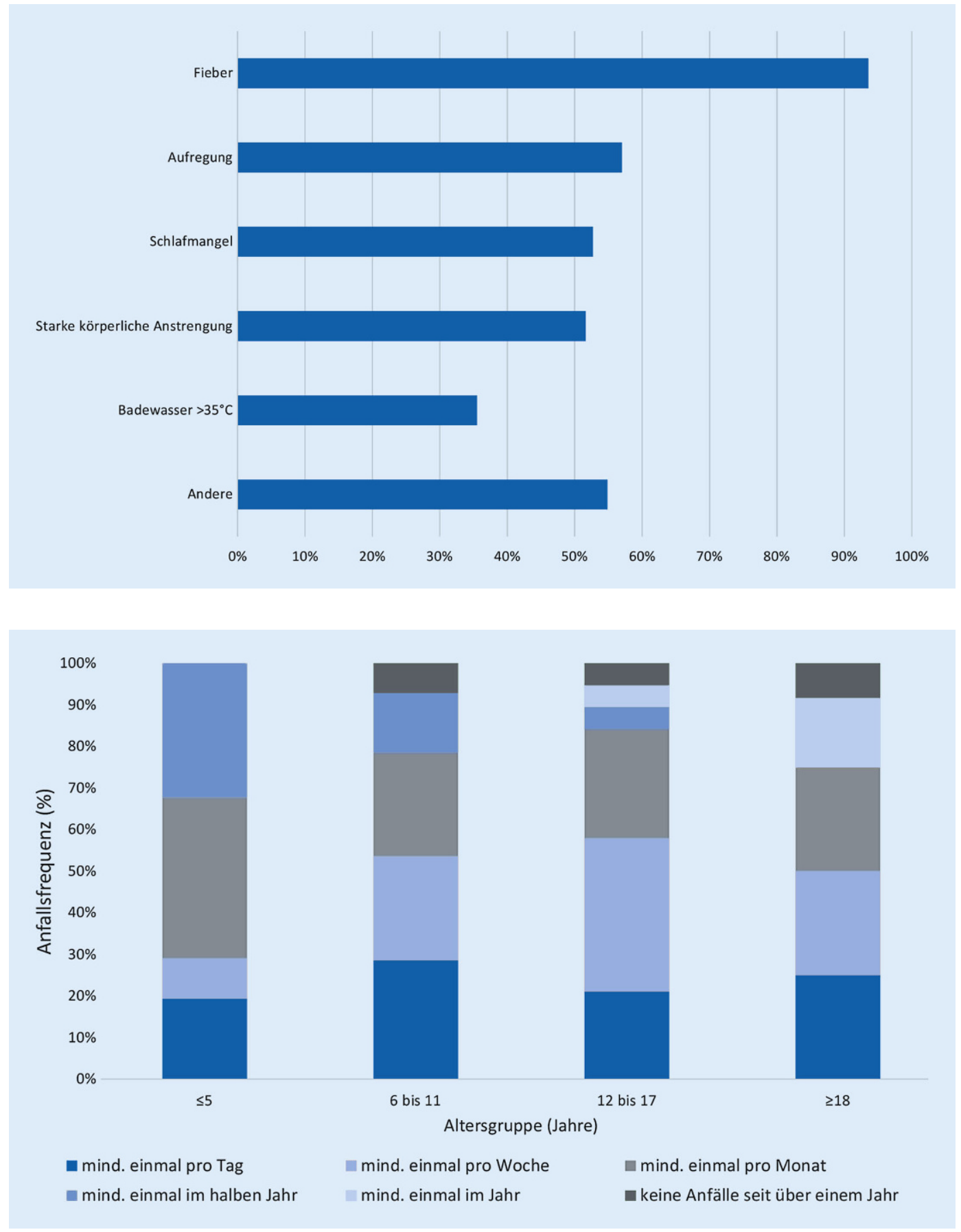

Abb. $3 \triangleleft$ Auslösende Faktoren für das Auftreten epileptischer Anfälle
Abb. $4<$ Anfallsfrequenz stratifiziert nach Altersgruppen bis zum Beginn einer antikonvulsiven Therapie, die nicht von einer Syndromdiagnose des DS abhängig ist.

Mutationen im SCN1A-Gen lagen bei $96 \%$ der Patienten mit DS $(n=89 / 93)$ vor. Neben dem typischen Alter bei Auftreten des ersten Anfalles (5,9 Monate) berichteten die Eltern auch über typische Auslöser für Anfälle. Fieber war mit 93,4\% der häufigste Trigger epileptischer Anfälle, gefolgt von Aufregung (56\%),
Schlafmangel (51,6\%), starker körperlicher Anstrengung (50,5\%) und einer Badewassertemperatur von mehr als $35^{\circ} \mathrm{C}$ (35,2\%). Weitere Ursachen wurden von insgesamt 53,8\% der Befragten angegeben, u. a. zählten das Auftreten von Hitzeperioden $(n=16 ; 17,2 \%)$, das Sehen in helles Licht oder das Betrachten bestimmter Muster $(n=7 ; 7,5 \%)$ sowie Wetterumschwünge $(n=6 ; 6,5 \%)$ dazu. $\mathrm{Zu}$ sammen mit Fieber wurden auch Infekte,
Impfungen und Zahnen als Auslöser benannt (• Abb. 3).

\section{Anfallshäufigkeit und Anfallssemiologie}

In den letzten 12 Monaten vor Studieneinschluss erlebten $95 \% \quad(n=88)$ der Patienten mindestens einen epileptischen Anfall. Die Angaben zur Anfallshäufigkeit aus dem retrospektiven 


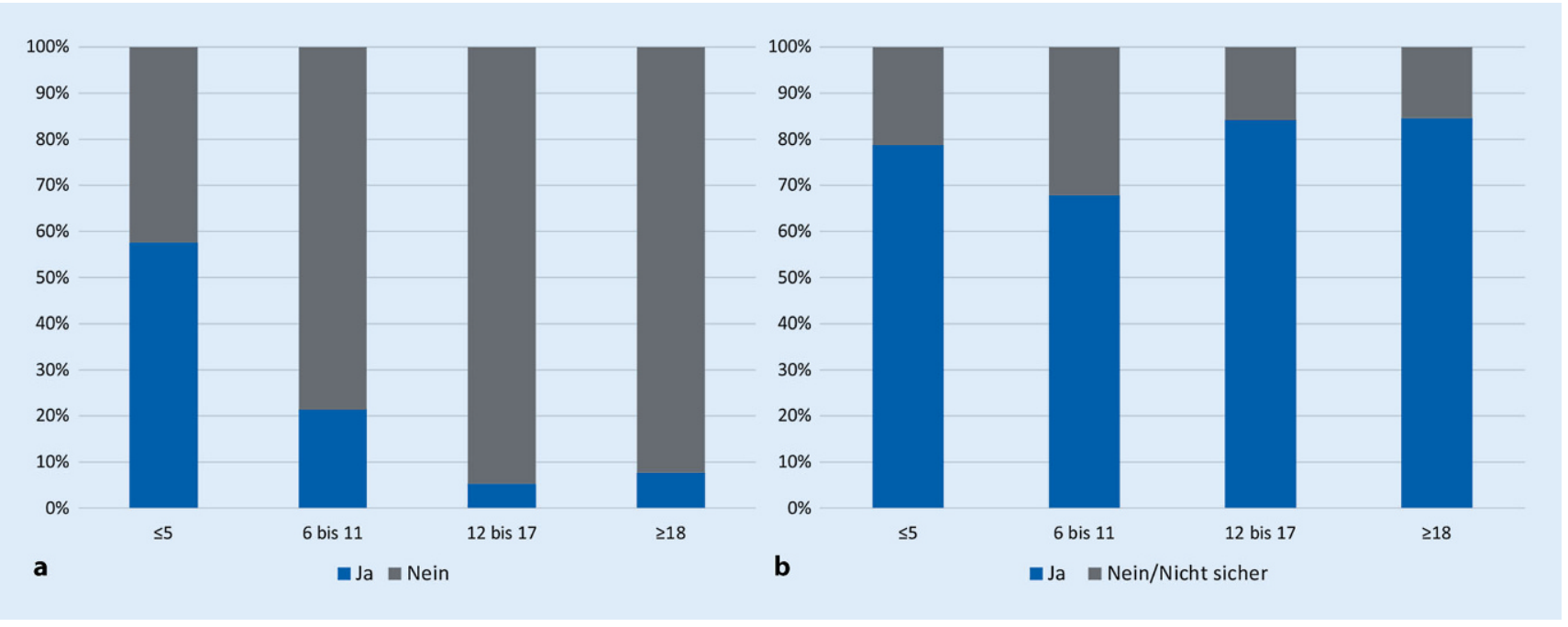

Abb. $5 \Delta$ a Der Anteil der Patienten mit Auftreten eines Status epilepticus innerhalb der letzten 12 Monate zeigte einen Rückgang mit zunehmendem Alter (in Jahren), bei Teenagern und Erwachsenen lag die Wahrscheinlichkeit bei unter $10 \%$. b Die Lebenszeitprävalenz liegt über alle Altersgruppen konstant bei $65-85 \%$

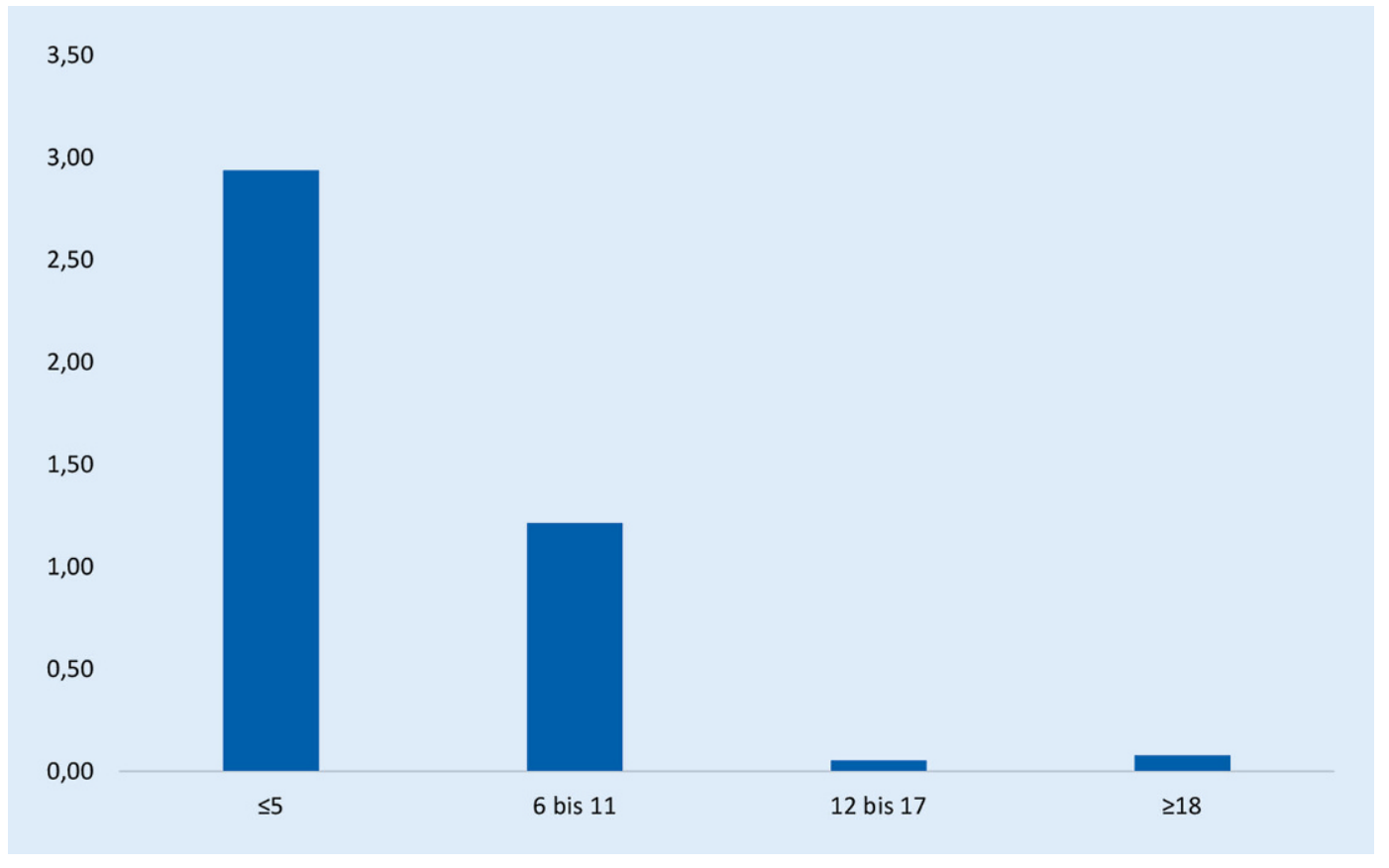

Abb. $6<$ Durchschnittliche Häufigkeit des Status epilepticus innerhalb der letzten 12 Monate, stratifiziert nach Altersgruppen (in Jahren)
Fragebogen und dem prospektiven Tagebuch waren vergleichbar. Die • Abb. 4 zeigt die Anfallshäufigkeit in Abhängigkeit vom Alter der Patienten mit DS. In der Kohorte hatte jeweils ein Viertel der Patienten mindestens 1 Anfall pro Tag, 1 oder mehr Anfälle pro Woche oder 1 oder mehr Anfälle im Verlauf eines Monats. Nur $4 \%$ der Patienten $(n=4)$ waren für mehr als 1 Jahr anfallsfrei, darunter befand sich keiner in der Altersgruppe von 5 Jahren oder jünger. Im Teenagerund Erwachsenenalter nahm die Anzahl der Patienten mit seltenen oder keinen Anfällen zu.

Bezüglich der Anfallssemiologie wurden bei $71 \%$ der Patienten während der prospektiven Beobachtungsperiode bilateral tonisch-klonische Anfälle (BTKA) berichtet. Bei Patienten mit mindestens 1 Anfall pro Jahr lag das kumulative Risiko bei $36 \%$ für mindestens 1 BTKA pro Woche und bei $63 \%$ für 1 im Monat. Myoklonien traten bei $46 \%$ der Patienten auf, hierbei wurde eine sehr hohe Frequenz angegeben. Bei 2 Patienten wurden in dem 3 Monate geführten Tagebuch über 3085 bzw. 7700 myoklonische Anfälle erfasst. Absencen erlitten $23 \%$, fokal tonische Anfälle $22 \%$, andere fokale Anfälle $21 \%$ und andere Anfallsarten 19\% der Patienten.

\section{Lebenszeitprävalenz und Häufigkeit des Status epilepticus}

Innerhalb der letzten 12 Monate war ein SE bei 26 Patienten (28\%) der Gesamtkohorte aufgetreten. Die $\bullet$ Abb. 5 a zeigt den 


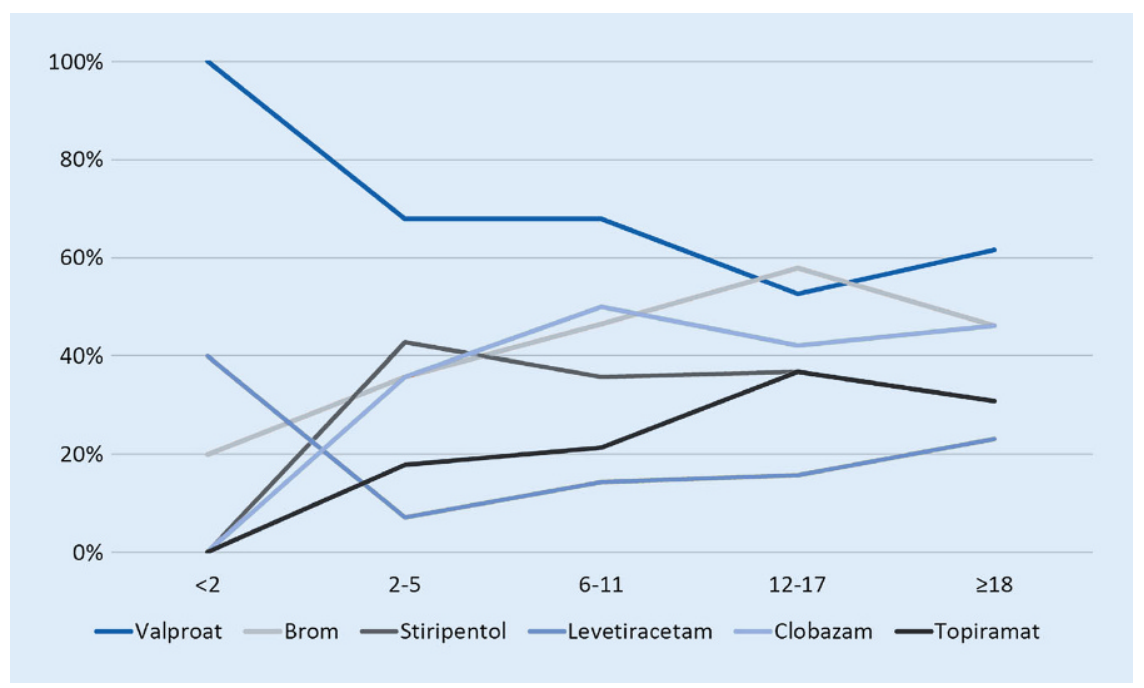

Abb. 7 \ Verordnungshäufigkeiten der sechs am häufigsten eingesetzten Antikonvulsiva, stratifiziert nach Altersgruppen (in Jahren)

Anteil der Patienten stratifiziert nach Altersgruppen, die einen SE in den letzten 12 Monaten erlebten. Dabei zeigt sich, dass ein SE bei Patienten im Alter von 5 Jahren oder jünger in über der Hälfte der Fälle aufgetreten war. Die Lebenszeitprävalenz für das Auftreten eines Status epilepticus (SE) wurde bei $77 \%$ der Patienten angegeben ( $\bullet$ Abb. $5 \mathbf{b}), 5 \%$ der Eltern waren sich nicht sicher, ob ein Status epilepticus bisher aufgetreten sei, und $17 \%$ der Eltern gaben an, dass nie ein SE aufgetreten sei. Die durchschnittliche jährliche Häufigkeit der Status epilepticus betrug 5 Episoden (Median 3; SA 5,1; SW 1-19). Die Abb. 6 zeigt diese Häufigkeit nach Altersgruppen stratifiziert. Insbesondere in den jüngeren Altersgruppen war die Frequenz deutlich höher. Ab dem Teenageralter trat ein SE nur noch bei wenigen Patienten mit einzelnen Episoden auf.

\section{Medikamentöse Therapie}

Nur 3\% der befragten Eltern gaben an, dass keine antikonvulsive Medikation eingenommen werde, die Mehrheit der DS-Patienten nahm 1 (11\%), 2 (30\%), $3(34 \%)$ oder $4(22 \%)$ antikonvulsive Medikamente (AED) ein. Details zur Dosierung und Kombinationsauswahl von Antikonvulsiva wurden von Schubert-Bast et al. berichtet [32]. $\mathrm{Zu}$ den am häufigsten verwendeten Antikonvulsiva gehören Valproat ( $n=61 ; 66 \%)$,
Kaliumbromid $(n=41 ; 44 \%)$, Clobazam $(n=38 ; 41 \%)$, Stiripentol $(n=31 ; 35 \%)$, Topiramat $(n=22 ; 24 \%)$ und Levetiracetam $(n=14 ; 15 \%)$ [32]. Die - Abb. 7 zeigt die Verordnungshäufigkeiten nach Altersgruppen aufgeteilt.

Natriumkanalblocker wie Lacosamid $(n=2)$, Lamotrigin $(n=1)$, Oxcarbazepin $(n=1)$ und Rufinamid $(n=1)$ wurden nur bei sehr wenigen Patienten eingesetzt [32]. Bezüglich des Verordnungsverhaltens zeigten sich keine wesentlichen Unterschiede im Vergleich zwischen Fragebogen und Tagebuch. Neben der antikonvulsiven Medikation setzten $16 \%(n=15)$ der Patienten spezifische Diäten, in der Regel eine ketogene Ernährungstherapie ein.

An weiteren langfristig eingenommenen Medikamenten wurde als Neuroleptikum Risperidon bei 2 Patienten $(2,2 \%)$ und als Psychostimulanzien jeweils 1-mal Lisdexamfetamin und Methylphenidat eingesetzt. Die Einnahme von Melatonin wurde bei 4 Patienten $(4,3 \%)$ berichtet. Eine Vitamin-D-Substitution erfolgte bei 35 Patienten (37,6\%).

In einem Zeitraum von 3 Monaten war bei zwei Dritteln der Patienten ein Einsatz von Bedarfsmedikation zur Notfallbehandlung von Anfällen notwendig. Am häufigsten kam bukkales Midazolam $(n=37 ; 42 \%)$ zum Einsatz, gefolgt von Diazepam rektal $(n=16 ; 17 \%)$ und Midazolam-Nasenspray $(n=5 ; 5 \%)$ [32].

\section{Soziodemografische Charakte- ristika und Lebensqualität}

Insgesamt leben $84 \%$ der Kinder in einem Haushalt mit 2 Elternteilen, wobei der Großteil der Eltern in Voll- oder Teilzeit berufstätig ist. Hiervon betrug der Anteil arbeitender Väter $82 \%$ und der arbeitender Mütter $56 \%$. Im Schulalter zwischen 5 und 17 Jahren besuchten $26 \%$ der Kinder und Jugendlichen eine Regelschule, $66 \%$ besuchten Spezialschulen oder einen geschützten Arbeitsplatz, $2 \%$ gingen einer Arbeit nach, und $6 \%$ befanden sich in anderen Einrichtungen.

Bei $78 \%$ der Patienten lag eine anerkannte Pflegestufe vor: $24 \%$ Pflegestufe 1, $27 \%$ Pflegestufe 2 und $27 \%$ Pflegestufe 3. Eltern von $11 \%$ der Kinder gaben an, keine Pflegestufe zuerkannt bekommen zu haben, aber eine zu benötigen. Dieser Anteil war bei den Patienten unter 2 Jahren mit $40 \%$ am höchsten; $11 \%$ gaben keine Pflegebedürftigkeit an.

Bei $89 \%$ der Patienten lag ein Behindertenausweis mit einem zuerkannten Grad der Behinderung (GdB) vor. Der durchschnittliche sozialrechtliche $\mathrm{GdB}$ der gesamten Kohorte betrug 80 von Hundert (v.H.; SA 21; SW 0-100). Es zeigte sich eine altersabhängige Zunahme mit durchschnittlichem GdB von 46 v.H. (SA 42; Median 70; SW 0-80) bei Kindern unter 2 Jahren, im Alter von 6 bis 11 Jahren lag der GdB bei durchschnittlich 81 v. H. (SA 31; Median 100; SW 0-100) und bei den 12- bis 17Jährigen bei 96 v. H. (SA 12,5; Median 100; SW 0-100). Bei den Erwachsenen erreichte der durchschnittliche GdB 97 v. H. (SA 11; Median 100; SW 60-100).

Innerhalb der Altersgruppen der 4- bis 6-Jährigen sowie der 7- bis 17Jährigen zeigte sich - erhoben mit einem standardisierten Instrument - eine unterdurchschnittliche Lebensqualität. Die mittlere Punktzahl betrug bei den 4- bis 6-Jährigen $(n=37)$ in der KiddyKINDL-Skala 65,98 (SA 15,35) und lag somit unter dem allgemeinen Durchschnitt der entsprechenden Altersklasse von 81,9 Punkten. Die Patienten im Alter von 7 bis 17 Jahren $(n=18)$ wurden anhand der Kid-KINDL-Skala beurteilt. Es zeigte sich wiederum ein unterdurchschnittlicher Wert von 54,75 (SD 12,9) im Vergleich zur entsprechenden Alters- 


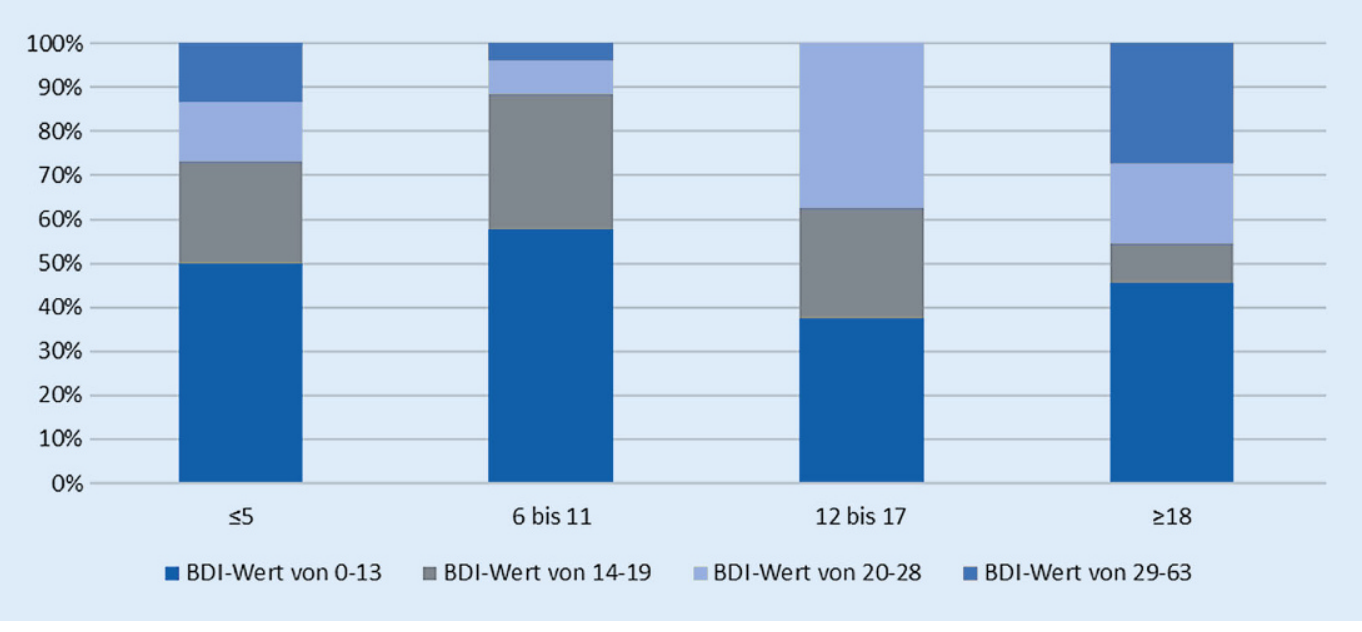

Abb. $8<$ Nach Altersgruppen der DS-Patienten (in Jahren) stratifizierte Angabe von Depressionssymptomen bei den Eltern bzw. Erziehungsberechtigten. Die Beck-Depressions Inventar(BDI) II-Werte von 0 bis 13 sprechen für keine, von 14 bis 19 für leichtgradige, von 20 bis 28 für moderate und von über 29 für schwergradige Depressionssymptome

gruppe (durchschnittliche Punktezahl 77,01) [31]. Die QoL der Kinder unter 4 Jahren und die der über 18-Jährigen wurde methodenbedingt nicht evaluiert.

Die Erhebung der Lebensqualität der Eltern bzw. Erziehungsberechtigten erfolgte mittels des generischen EQ-5D3L. Der Durchschnittswert lag bei 0,9 (SA 0,18; SW 0,3-1) und somit deckungsgleich im Vergleich mit der erwachsenen Bevölkerung in Deutschland. Die Messung der durchschnittlichen Lebensqualität auf der visuellen Analogskala („Thermometer von 0 bis 100“; EQ-5D-3L VAS) ergab durchschnittlich 71,3 (SA 17,95; SW 19-100) und lag damit unterhalb des Durchschnittswertes von 77,3 für die Allgemeinbevölkerung.

Der BDI-II wurde herangezogen, um Depressionssymptome zu erfassen, hierbei zeigte sich ein Durchschnittswert von 15,4 Punkten (SA 9,7; SW 0-43), wobei $46 \%$ der Elternteile den Cut-off-Wert von 14 und mehr Punkten überschritten. Bei $22 \%(n=20)$ entsprach dies leichten Depressionssymptomen, bei $15 \%(n=13)$ moderaten und bei $9 \%(n=8)$ schweren Depressionssymptomen. Dabei misst der BDI-II nur Depressionssymptome und erlaubt keinen Rückschluss auf die klinisch zu stellende Diagnose einer manifesten Depression, zudem wurden keine Verlaufswerte erhoben, und es erfolgte keine Befragung bezüglich einer manifesten Depression in der Vorgeschichte. Die Abb. 8 zeigt die Verteilung der BDIWerte, stratifiziert nach Altersgruppen.

\section{Direkte und indirekte Kosten}

Insgesamt berichteten $52 \%(n=48)$ der Eltern über mindestens eine stationäre Krankenhausaufnahme innerhalb der letzten 12 Monate. In diesem Zeitraum mussten $47 \%(n=44)$ mindestens 1-mal den Rettungsdienst verständigen. Durchschnittlich wurde der Rettungsdienst 4,23-mal (SA 4,86; SW 1-30) gerufen, und die Patienten wurden im Schnitt 4,27-mal (SA 5,26; SW1-35) hospitalisiert. In der Kohorte der stationär aufgenommenen Patienten betrug die durchschnittliche Anzahl an Krankenhaustagen 25,6 Tage (SA 39,6; SW 1-200), davon wurden 5,5 Tage (SA 5,94; SW 1-23) auf einer Intensivstation verbracht [31].

Die gesundheitsökonomische Auswertung des Ressourcenverbrauches über 3 Monate bei Patienten mit DS erbrachte im Mittel direkte Kosten in Höhe von $6043 €$ (SA 5947; SW148-30.696€), dies entspricht einem durchschnittlichen Jahreswert von ca. 25.000€ [31]. Die Kosten für Krankenhausbehandlungen machten dabei mit $1702 €$ für 3 Monate (SA 4315; SW 0-20.736€) den größten Teil $(28,2 \%)$ aus, gefolgt von Kosten für Pflegeleistungen mit $1130 €$ (SA $805 €$; SW 0-2184€; 18,7\%), Kosten für AED mit $892 €$ (SA 1018; SW $0-4779 € ; 14,8 \%)$ und Kosten für Therapien mit 559€ (SA 503; SW 0-2152,26€; 8,3\%). Einen Überblick über die direkten Kosten verschafft - Abb. 9a.

Die Tab. 1 zeigt eine detaillierte Auswertung der ambulanten Versor- gung im ärztlichen Bereich sowie durch weitere medizinische Fachkräfte und durch nichtmedikamentöse Therapien. Deutlich zeigt sich der hohe Bedarf an Physio- (55\%) sowie Ergotherapie (44\%) und Logopädie (43\%) bei dem Großteil der Befragten mit einer medianen Therapiedichte von 10 bis 12 Sitzungen pro 3 Monate. Auffällig ist die hohe Notwendigkeit der Zuzahlung durch die Eltern bzw. Erziehungsberechtigten. Insgesamt sind dies 520€ Eigenanteil (SA 1329; SW 0-8360€) für medizinische Ausgaben sowie weitere Ausgaben in Höhe von 624€ (SA 2402; SW 0-20.350€) in 3 Monaten.

Die indirekten Kosten entstanden zum Großteil durch das Aufgeben der Berufstätigkeit bzw. durch eine Arbeitszeitreduktion. Einen Überblick bietet - Abb. 9b. Die Veränderungen der Arbeitssituation wurden für Mütter und Väter der DS-Patienten getrennt ausgewertet. In dem Betrachtungszeitraum von 3 Monaten betrugen die mittleren indirekten Kosten $4399 €$ (SA 4990; SW 0-20.191€) bei den Müttern und $391 €$ (SD 1352; SW 0-10.165€) bei den Vätern, jeweils als Durchschnittswert, der auf die gesamte Kohorte berechnet wurde [31].

Von 93 Müttern gaben 29 ihre Arbeit aufgrund des DS ihres Kindes auf, wodurch indirekte Kosten in Höhe von $3170 €$ (SA $4734 €$; SW 0-10.165€) bezogen auf 3 Monate entstanden; $27 \mathrm{der}$ 93 Mütter reduzierten ihre Arbeitsstunden, die so entstandenen Kosten betrugen im Schnitt $732 €$ (SA 1522; SW 


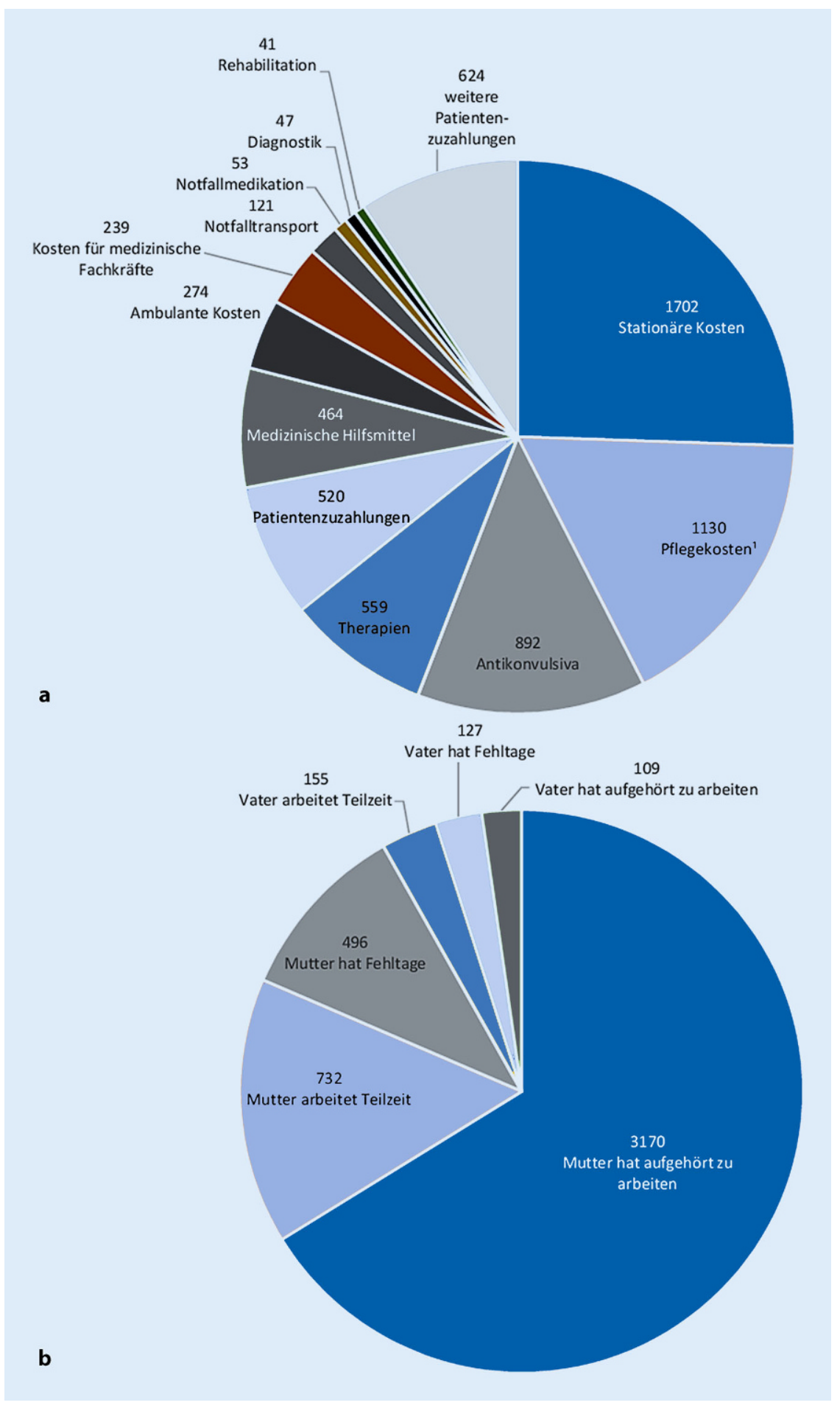

Abb. 9 ム Direkte und indirekte Kosten innerhalb der letzten 3 Monate. a Direkte Kosten über 3 Monate $(€) .{ }^{1}$ Pflegeleistung wurden an Hand der Pflegestufen bei angenommener Erbringung durch Familienangehörige berechnet. b Indirekte Kosten über drei 3 Monate $(€)$. (Adaptiert nach [31])

0-10.030€), und 37 versäumten Arbeitstage, wodurch indirekte Kosten in Höhe von $496 €$ entstanden (SA 1595; SW $0-10.026 €)$. Bei den Vätern entstanden die größten indirekten Kosten in dem 3monatigem Betrachtungszeitraum durch die Reduktion der Arbeitszeit, diese betrugen im Schnitt $155 €$ (SD 635; SW 0-3343€) und betraf 6 der 93 Väter; 25 Väter versäumten Arbeitstage, wobei Kosten in Höhe von $127 €$ (SD 374; SW 0-3008€) entstanden, und 1 Vater gab seine Arbeit auf, hieraus errechnen sich durchschnittliche indirekte Kosten in Höhe von $109 €$.

\section{Diskussion}

Die vorliegende Studie gibt einen Überblick über die aktuelle Versorgungssituation (Stand 2017/18) von Patienten mit Dravet-Syndrom und deren Eltern. Anhand einer prospektiven, multizentrischen Erhebung wurden Daten einer großen Kohorte von 93 Patienten mit DS deutschlandweit erhoben. Es zeigt sich eindrücklich, dass die Dauer bis zur Diagnosestellung in den letzten beiden Jahrzehnten deutlich reduziert werden konnte. Die eingeschlossenen Patienten zeigen den typischen Krankheitsbeginn um den 6. Lebensmonat. Dabei benennen die Eltern typische Anfallsauslöser wie Fieber, Aufregung, Schlafmangel und starke körperliche Anstrengung. Ebenfalls typisch sind die große Variabilität der berichteten Anfallstypen, sowie das häufige Auftreten eines Status epilepticus in den ersten Lebensjahren.

Bezüglich der sozioökonomischen Implikationen des DS auf Patienten und ihre Eltern bzw. Erziehungsberechtigten zeigen sich hohe direkte und indirekte Kosten. Bislang liegen aus Europa nur zwei weitere Studien zu dieser Fragestellung vor. Eine Pilotstudie an 13 Patienten mit DS aus dem Norddeutschen Epilepsiezentrum stellte die häufigen Krankenhausbehandlungen als Hauptkomponente der direkten Kosten dar, aber auch die Möglichkeit, diese durch den Einsatz neuer zielgerichteter Therapien zu senken [33]. In dieser Pilotstudie zeigte sich ein deutlicher Rückgang der Krankenhausbehandlungen nach der Einführung von Stiripentol (Auswertezeitraum 2007 bis 2010) [33]. Lagae et al. führten eine internetbasierte Befragung von 584 Eltern mit von DS betroffenen Kindern in 10 Sprachen in Europa durch, dabei kamen $12 \%$ der Teilnehmer aus Deutschland [34]. Diese Befragung ist mit unserer Studie hinsichtlich demografischer und klinischer Basisdaten gut vergleichbar, es zeigt sich ebenfalls eine hohe Anzahl von Rettungsdiensteinsätzen und Krankenhausbehandlungen [34]. Durch die 
Tab. 1 Kosten, Häufigkeiten und Zuzahlungen bei Arztbesuchen und Therapien innerhalb von 3 Monaten

\begin{tabular}{|c|c|c|c|c|c|c|c|c|}
\hline \multirow[t]{2}{*}{$\begin{array}{l}\text { Arztbesuche/ } \\
\text { Behandlungen }\end{array}$} & \multirow[t]{2}{*}{$\%$} & \multicolumn{2}{|c|}{$\begin{array}{l}\text { Häufigkeit in den letzten } \\
3 \text { Monaten }\end{array}$} & \multicolumn{2}{|l|}{ Kosten (€) } & \multicolumn{3}{|c|}{$\begin{array}{l}\text { Zuzahlungen ( } € \text { ) } \\
\text { (Bei denjenigen, die Zuzahlungen angaben) }\end{array}$} \\
\hline & & $\begin{array}{l}\text { Mittelwert } \\
\text { (SD) }\end{array}$ & Median & Mittelwert (SD) & Median & $\begin{array}{l}\% \text { gaben Zuzah- } \\
\text { lungen an }\end{array}$ & Mittelwert (SD) & Median \\
\hline Neurologe/-in & 78 & $2,57(2,16)$ & 2,0 & $121,0(101,5)$ & 94,0 & 10 & $94,9(99,9)$ & 50,0 \\
\hline Allgemeinmediziner/-in & 60 & $3,20(2,89)$ & 2,0 & $67,4(61,0)$ & 42,2 & 3 & $10,0(0,0)$ & 10,0 \\
\hline Orthopäde/-in & 30 & $1,43(1,20)$ & 1,0 & $38,2(32,0)$ & 26,7 & 4 & $40,0(42,4)$ & 25,0 \\
\hline $\begin{array}{l}\text { Kinder- und Jugendpsychia- } \\
\text { ter/-in }\end{array}$ & 5 & $2,40(1,14)$ & 2,0 & $196,9(93,5)$ & 164,1 & 2 & $45,0(7,1)$ & 45,0 \\
\hline Radiologe/-in & 43 & $2,00(2,00)$ & 1,0 & $45,2(45,2)$ & 22,6 & 0 & Keine Angabe & \\
\hline Zahnarzt/-ärztin & 31 & $1,28(0,70)$ & 1,0 & $74,9(41,2)$ & 58,7 & 4 & $35,0(37,9)$ & 20,0 \\
\hline Alternativmediziner/-in & 3 & $8,00(6,56)$ & 9,0 & $419,7(344,0)$ & 472,1 & 2 & $310,0(120,2)$ & 310,0 \\
\hline Homöopath/-in & 16 & $2,13(1,19)$ & 2,0 & $111,9(62,3)$ & 104,9 & 13 & $136,3(113,5)$ & 90,0 \\
\hline Ernährungsberater/-in & 54 & $2,00(2,24)$ & 1,0 & $42,2(47,1)$ & 21,1 & 1 & $394,0(0,0)$ & 394,0 \\
\hline Sonstige Fachkräfte & 29 & $1,78(1,69)$ & 1,0 & $83,5(93,1)$ & 54,5 & 4 & $92,3(69,5)$ & 95,5 \\
\hline Physiotherapie & 51 & $13,68(8,86)$ & 12,0 & $236,0(152,9)$ & 207,0 & 8 & $78,7(71,5)$ & 48,0 \\
\hline Logopädie & 40 & $9,35(4,02)$ & 10,0 & $379,2(163,1)$ & 405,5 & 3 & $28,3(27,5)$ & 15,0 \\
\hline Ergotherapie & 41 & $10,58(3,63)$ & 12,0 & $416,9(143,0)$ & 472,9 & 2 & $270,0(212,1)$ & 270,0 \\
\hline Akupunktur & 2 & $6,00(4,24)$ & 6,0 & $300,5(212,5)$ & 300,5 & 2 & $172,5(74,3)$ & 172,5 \\
\hline Hippotherapie & 13 & $8,67(4,38)$ & 10,0 & $292,8(147,9)$ & 337,8 & 12 & $243,3(178,7)$ & 320,0 \\
\hline Sonstige Therapien & 27 & $10,36(6,55)$ & 10,0 & $286,9(172,2)$ & 258,8 & 17 & $175,3(202,5)$ & 110,0 \\
\hline
\end{tabular}

gezielte sozioökonomische Ausrichtung unserer Studie konnten wir den hohen Ressourceneinsatz und die Notwendigkeit kontinuierlicher Therapien (vgl. - Tab. 1) darstellen. Problematisch ist die hohe finanzielle Belastung der Eltern bzw. Erziehungsberechtigten durch $\mathrm{Zu}$ zahlungen für medizinische Therapien und weitere nichtmedizinische Kostenfaktoren.

Um diesen Aspekt zu verdeutlichen, erfolgte eine vergleichende Auswertung [35] zwischen unserer Dravet-Kohorte und nach Alter und Geschlecht abgestimmten Epilepsiepatienten mit therapierefraktärem Verlauf, jedoch ohne Vorliegen einer epileptischen Enzephalopathie und mit Epilepsiepatienten in Anfallsremission. Hierfür wurden Studiendaten aus früher durchgeführten gesundheitsökonomischen Evaluationen in Hessen und Schleswig-Holstein $[25,36]$ verwendet. Bezüglich der Anwendung von Therapien wie Logopädie, Physiotherapie und Ergotherapie zeigte sich bei Patienten mit einem DS ein deutlich erhöhter Bedarf im Vergleich zu Patienten mit anderer therapierefraktärer Epilepsie oder zu solchen in Anfallsremission. Ähnliche Ergebnisse erzielt die DISCUSS-Umfrage [37], welche Physiotherapie als den größ- ten nicht anfallsassoziierten Teil der Kosten bei DS bestätigte. Die Auswirkungen der häufigen Episoden eines Status epilepticus sind nicht unerheblich, da diese mit erhöhten Kosten, langen Krankenhausaufenthalten, verminderter Lebensqualität und einer erhöhten Mortalität verbunden sind [38, 39, 48].

Die sozialen Auswirkungen des DS spiegeln sich in den indirekten Kosten in Höhe von $19.200 €$ pro Jahr wider und sind v. a. in der Niederlegung der Berufstätigkeit sowie der Arbeitszeitreduktion vorrangig der Mütter begründet. Dass die Hauptlast der Betreuung von Kindern mit Epilepsie von Müttern getragen wird, zeigte bereits die Studie zu Kindern und Jugendlichen mit Epilepsie von Riechmann et al. [25]. Die dort angegebenen indirekten Kosten von $5200 €$ pro Jahr liegen deutlich unterhalb der Kosten beim DS.

Bei den Patienten mit DS ergibt sich eine verringerte Lebensqualität im Vergleich zur Durchschnittsbevölkerung. Dies wurde bereits in anderen Studien bestätigt [34, 40, 50], wobei der spezifische KINDL-Fragebogen eine detailliertere Auswertung erlaubt. Insbesondere in den Bereichen „Schule“, „Freunde“ und „Selbstbewusstsein“ erreichen Kinder und Jugendliche mit DS vergleichsweise besonders niedrige Werte [27]. Dies lässt den Rückschluss zu, dass bei der Integration von Kindern mit DS in unsere Gesellschaft noch Verbesserungsbedarf besteht.

Die Auswirkungen des DS auf Eltern und Erziehungsberechtigte wurde in dieser Studie mittels der Erhebung von Depressionssymptomen (BDI-II) erfasst. Ein hoher Anteil von $45 \%$ gab Depressionssymptome an. Diese waren vergleichbar mit den Werten von Eltern anderer therapierefraktärer Epilepsiepatienten [41, 42]. In einer Umfrage der Dravet Syndrome Foundation mittels offener Befragung von 256 Eltern (70\% aus den USA) stellten Villas et al. fest, dass etwa $60 \%$ der Betreuenden von DS-Patienten an depressiven Symptomen litten [43]. Eine Auswirkung auf die rein gesundheitsbezogene, generische Lebensqualität, gemessen anhand der Lebensqualitätsfragebögen wie den EQ5D-3L, besteht nicht, wobei die Eltern auf der visuellen Thermometerskala von 0 bis 100 ihre Lebensqualität gegenüber der Gesamtbevölkerung als geringer einschätzen. Insgesamt schlussfolgert ein Review zur Auswirkung des DS auf Familie und Betreuende, dass der 


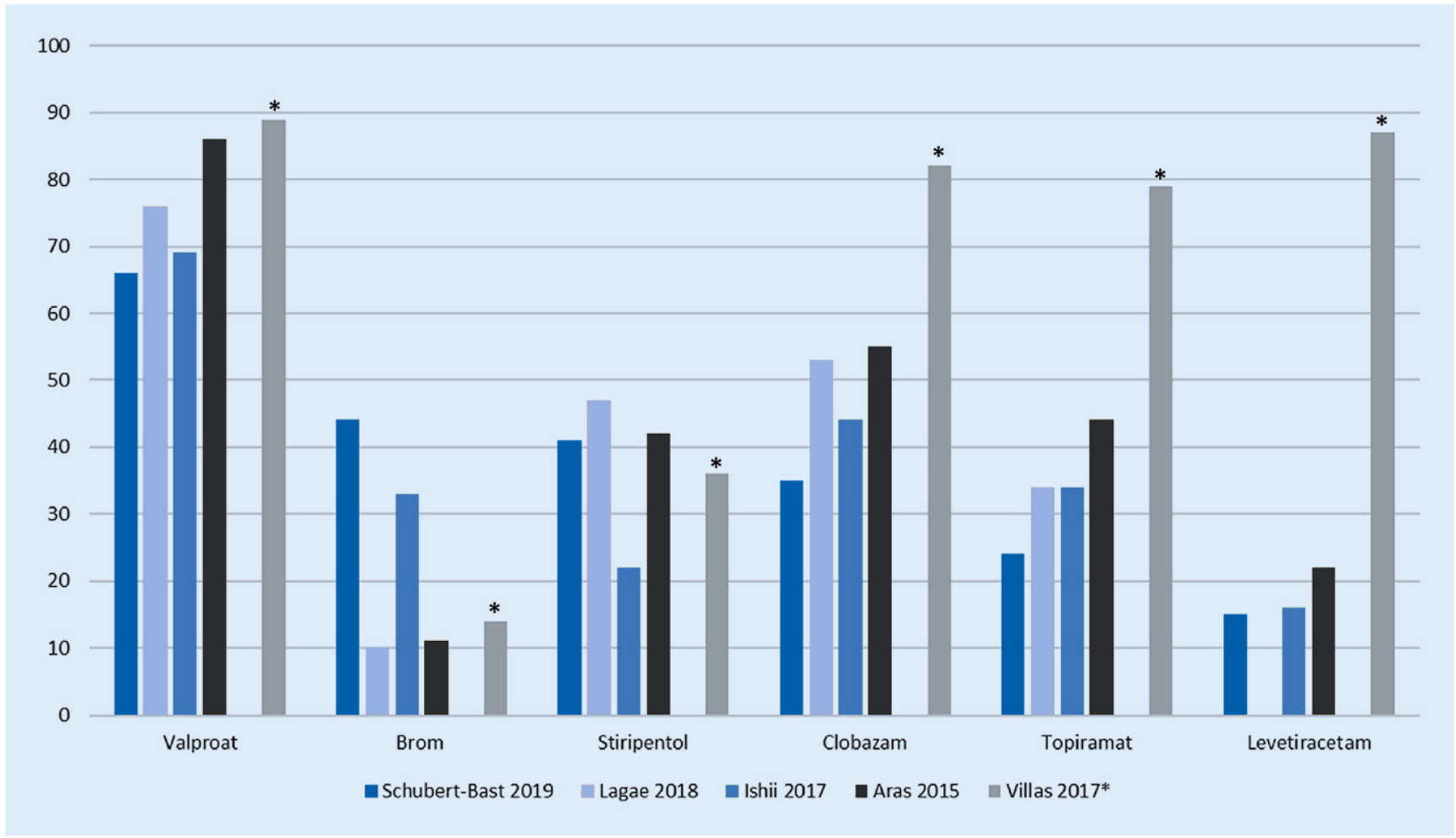

Abb. $10 \Delta$ Einsatz von AEDs beim Dravet-Syndrom im Vergleich rezent publizierter Studien, Häufigkeiten aktuell eingenommener AEDs, entnommen aus Schubert-Bast et al. [32], Lagae et al. [34], Ishii et al. [45], Aras et al. [1] und Villas et al. [43]. ${ }^{*}$ bei Villas et al. sind alle bereits eingesetzten AEDs (Lebenszeitprävalenz) dargestellt

Großteil der Implikationen der Pflege und Betreuung eines Kindes mit therapierefraktärer Epilepsie bzw. einem DS weiterhin unklar bleibt [44].

Die in unserer Studie eingesetzten Antikonvulsiva korrelieren gut mit dem auch in anderen Studien berichteten häufigen Einsatz von Valproat, Clobazam, Topiramat, Stiripentol und Levetiracetam [1]. Eine vergleichende Darstellung des Einsatzes von Antikonvulsiva in den einzelnen Studien ist in 0 Abb. 10 dargestellt. Zudem berichteten die Eltern regelhaft über den Einsatz von ketogenen Ernährungstherapien zur Anfallsreduktion [32].

Die Häufigkeit des Einsatzes von Kaliumbromid unterscheidet sich zwischen den einzelnen Studien und liegt auch an der unterschiedlichen regionalen Verfügbarkeit dieses lang etablierten Antikonvulsivums begründet. Insgesamt $44 \%$ unserer Kohorte werden mit Kaliumbromid behandelt, auch andere Erhebungen aus Deutschland belegen die gute Wirksamkeit von Kaliumbromid [46, 47]. Neben Deutschland wurde Kaliumbromid auch in Japan häufig eingesetzt [45]. Die Um- frage der Dravet Syndrome Foundation bezüglich aller bislang eingesetzten Antikonvulsiva belegt, dass Levetiracetam, Topiramat und Clobazam häufig eingesetzt wurden [43], was sich jedoch nicht in den aktuell eingenommenen Medikamenten widerspiegelt, welche in den anderen Studien aus Europa und Japan berichtet werden $[1,37,45]$. Der hohen Diskrepanz zwischen dem aktuellen Einsatz und der Lebenszeitprävalenz liegt wahrscheinlich eine geringe Wirksamkeit von Levetiracetam beim DS zugrunde. Die medikamentöse Therapie bleibt trotz der vielen inzwischen verfügbaren $A E D s$ auf einige wenige häufig eingesetzte Antikonvulsiva beschränkt. Wie sich das Verordnungsverhalten nach der Einführung von Cannabidiol und Fenfluramin verändern wird, bleibt spekulativ und die Antwort zukünftigen Studien vorbehalten.

Hinsichtlich des Einsatzes der Notfallmedikamente gibt es keine Vergleichsdaten in Europa. In der Befragung von Villas et al., welche ebenfalls zu $18 \%$ europäische Patienten mit DS einschloss, wurden Diazepam und Midazolam mit 98 \% bzw.
$68 \%$ am häufigsten eingesetzt [43]. Dies ist annähernd vergleichbar mit den hier erhobenen Daten, wobei bukkales Midazolam mit $40 \%$ und Diazepam mit 20\% am häufigsten genannt wurden.

$\mathrm{Zu}$ den Limitationen der Studie gehört die teilweise retrospektive Erfassung durch den Fragebogen, wobei eine gute Übereinstimmung mit dem Tagebuch gezeigt werden konnte. Zudem erfolgte keine zentrale Schulung der Eltern in der Anfallsdokumentation für das Tagebuch, sodass wir nicht ausschließen können, dass die semiologische Einordnung der Anfälle nicht übereinstimmend durch alle Eltern erfolgte. Die Fragebögen wurden über den Dravet-Syndrom e. V. sowie zahlreiche Neuropädiater und Epilepsiezentren verteilt, dennoch können wir nicht ausschließen, dass eine Verzerrung vorliegt mit mehrheitlichem Einschluss v. a. von Eltern aus dem DravetSyndrom e.V.

\section{Schlussfolgerung}

Das Dravet-Syndrom ist mit häufigen, oft therapierefraktären epileptischen Anfäl- 
len und Status epilepticus vergesellschaftet. Unsere Studie belegt die erhebliche Krankheitslast und die damit verbundenen Einschränkungen in der Lebensqualität sowie die hohen direkten und indirekten Kosten. Die Belastung ist dabei im Vergleich zu anderen therapierefraktären Epilepsien ohne Enzephalopathie nochmals höher. Um eine Verbesserung der Lebensqualität bei Patienten mit DS und deren Eltern zu erreichen, bedarf es neuer Therapie- und Versorgungskonzepte sowie einer verbesserten Unterstützung der Betreuenden von Patienten mit einem DS.

\section{Korrespondenzadresse}

\section{Prof. Dr. Adam Strzelczyk}

Epilepsiezentrum Frankfurt Rhein-Main und Zentrum für Neurologie und Neurochirurgie, Goethe-Universität Frankfurt

Schleusenweg 2-16, Haus 95, 60528 Frankfurt am Main, Deutschland

strzelczyk@med.uni-frankfurt.de

Danksagung. Die Autoren bedanken sich bei allen Patienten und deren Betreuenden, die zu dieser Studie beigetragen haben. Für die sehr engagierte Unterstützung bei der Patientenrekrutierung danken wir der deutschen Selbsthilfegruppe DravetSyndrom e. V.

Förderung. Diese Studie wurde finanziell unterstützt durch Zogenix International Limited.

\section{Einhaltung ethischer Richtlinien}

Interessenkonflikt. S. Schubert-Bast erhielt Beratungs- oder Referentenhonorare von UCB, Desitin Arzneimittel, Novartis, Zogenix, LivaNova, und Eisai. R. Trollmann berichtet über Referentenhonorare von Novartis, Desitin Arzneimittel, UCB Pharma und Pfizer. T. Bast erhielt Beratungs- oder Referentenhonorare von Bial, Biocodex, Eisai, Desitin Arzneimittel, GWPharmaceuticals, Nutricia, Shire, UCB Pharma, Viropharma und Zogenix. A. Wiemer-Kruel erhielt Beratungs- oder Referentenhonorare von Desitin Arzneimittel, Dr. Schär, Novartis, Nutricia, Vitaflo, UCB Pharma. G. Kurlemann erhielt Beratungs- oder Referentenhonorare von UCB Pharma, Desitin Arzneimittel, Zogenix, LivaNova, Eisai, GW Pharma, Bial, Dibropharma, Novartis, Biogen, Actelion. G. Kluger erhielt Beratungs- oder Referentenhonorare von Desitin Arzneimittel, Eisai und Zogenix. D. Macdonald, J. Carroll und C. Pritchard sind bei Wickenstones Ltd angestellt und erhalten Unterstützung von Zogenix International Limited für diese Studie. K.M. Klein erhielt Beratungs- oder Referentenhonorare von UCB Pharma, Novartis Pharma AG, Eisai und GW Pharmaceuticals. F. Rosenow: Beratungs-, Referentenhonorare und/oder Unterstützung für Forschungsvorhaben von Eisai, UCB Pharma, Desitin Arzneimittel, Novartis, GW Pharma, Medtronic, Cerbomed and Shire, der Europäischen Union, der Deutschen Forschungsgemeinschaft, vom Land
Hessen über das LOEWE-Programm und vom DetlevWrobel-Fonds für Epilepsieforschung. A. Strzelczyk erhielt Beratungs-, Referentenhonorare und/oder Unterstützung für Forschungsvorhaben von Desitin Arzneimittel, Eisai, GW Pharma, LivaNova, Medtronic Sage Therapeutics, UCB Pharma und Zogenix. L. Kay erhielt Reisekostenunterstützung von Eisai und UCB. M. Kalski, M. Kieslich, A.-C. Leyer, T. Polster, A. Herting, T. Mayer, B.A. Neubauer, U. Bettendorf, S. von Spiczak, M. Wolff und J. Irwin geben an, dass kein Interessenkonflikt besteht.

Alle beschriebenen Untersuchungen am Menschen oder an menschlichem Gewebe wurden mit Zustimmung der zuständigen Ethikkommission, im Einklang mit nationalem Recht sowie gemäß der Deklaration von Helsinki von 1975 (in der aktuellen, überarbeiteten Fassung) durchgeführt. Von allen beteiligten Patienten liegt eine Einverständniserklärung vor.

Open Access. Dieser Artikel wird unter der Creative Commons Namensnennung 4.0 International Lizenz (http://creativecommons.org/licenses/by/4.0/deed. de) veröffentlicht, welche die Nutzung, Vervielfältigung, Bearbeitung, Verbreitung und Wiedergabe in jeglichem Medium und Format erlaubt, sofern Sie den/die ursprünglichen Autor(en) und die Quelle ordnungsgemäß nennen, einen Linkzur Creative Commons Lizenz beifügen und angeben, ob Änderungen vorgenommen wurden.

\section{Literatur}

1. Aras LM, Isla J, Mingorance-Le Meur A (2015) The European patient with Dravet syndrome: results from a parent-reported survey on antiepileptic drug use in the European population with Drave syndrome. Epilepsy Behav 44:104-109

2. Dravet C (2011) The core Dravet syndrome phenotype. Epilepsia 52(Suppl 2):3-9

3. Genton P, Velizarova R, Dravet C (2011) Dravet syndrome: the long-term outcome. Epilepsia 52(Suppl 2):44-49

4. Gataullina S, Dulac O (2017) From genotype to phenotype in Dravet disease. Seizure 44:58-64

5. Rilstone JJ, Coelho FM, Minassian BA, Andrade DM (2012) Dravet syndrome: seizure control and gait in adults with different SCN1A mutations. Epilepsia 53(8):1421-1428

6. Brunklaus A, Ellis R, Reavey E, Forbes GH, Zuberi SM (2012) Prognostic, clinical and demographic features in SCN1A mutation-positive Dravet syndrome. Brain 135(Pt 8):2329-2336

7. Rosander C, HallbookT (2015) Dravet syndrome in Sweden: a population-based study. Dev Med Child Neurol 57(7):628-633

8. Bayat A, Hjalgrim H, Moller RS (2015) The incidence of SCN1A-related Dravet syndrome in Denmark is 1:22,000: a population-based study from 2004 to 2009. Epilepsia 56(4):e36-e39

9. Wu YW, Sullivan J, McDaniel SS, Meisler MH, Walsh EM, Li SX, Kuzniewicz MW (2015) Incidence of Dravet syndrome in a US population. Pediatrics 136(5):e1310-1315

10. Auvin S, Irwin J, Abi-Aad P, Battersby A (2018) The problem of rarity: estimation of prevalence in rare disease. Value Health 21(5):501-507

11. Claes L, Del-Favero J, Ceulemans B, Lagae L, Van Broeckhoven C, De Jonghe P (2001) De novo mutations in the sodium-channel gene SCN1A cause severe myoclonic epilepsy of infancy. Am J Hum Genet 68(6):1327-1332
12. Cooper MS, McIntosh A, Crompton DE, McMahon JM, Schneider A, Farrell K, Ganesan V, Gill D, Kivity S, Lerman-Sagie T et al (2016) Mortality in Dravet syndrome. Epilepsy Res 128:43-47

13. Kassai B, Chiron C, Augier S, Cucherat M, Rey E, Gueyffier F, Guerrini R, Vincent J, Dulac O, Pons G (2008) Severe myoclonic epilepsy in infancy: a systematic review and a meta-analysis of individual patient data. Epilepsia 49(2):343-348

14. Von Spiczak S, Stephani U (2019) Therapie des Dravet-Syndroms.ZEpileptol 32(2):107-115

15. Wirrell EC, Laux L, Donner E, Jette N, Knupp K, Meskis MA, Miller I, Sullivan J, Welborn M, Berg AT (2017) Optimizing the diagnosis and management of Dravet syndrome: recommendations from a north American consensus panel. Pediatr Neurol 68:18-34e13

16. Tian $X$, Chen J, Zhang J, Yang $X$, Ji T, Zhang $Y$, Wu Y, Fang F, Wu X, Zhang Y (2019) The efficacy of Ketogenic diet in 60 Chinese patients with Dravet syndrome. Front Neurol 10:625

17. Devinsky O, Cross JH, Laux L, Marsh E, Miller I, Nabbout $R$, Scheffer IE, Thiele EA, Wright $S$ (2017) Trial of Cannabidiol for drug-resistant seizures in the Dravet syndrome. N Engl J Med 376(21):2011-2020

18. Ceulemans B, Boel M, Leyssens K, Van Rossem C, Neels P, Jorens PG, Lagae L (2012) Successful use of fenfluramine as an add-on treatment for Dravet syndrome. Epilepsia 53(7):1131-1139

19. Schoonjans A, Paelinck BP, Marchau F, Gunning B, Gammaitoni A, Galer BS, Lagae L, Ceulemans B (2017) Low-dose fenfluramine significantly reduces seizure frequency in Dravet syndrome: a prospective study of a new cohort of patients. Eur JNeurol 24(2):309-314

20. Polster T (2019) Individualized treatment approaches: Fenfluramine, a novel antiepileptic medication for the treatment of seizures in Dravet syndrome. Epilepsy Behav 91:99-102

21. Fisher RS, Cross JH, French JA, Higurashi N, Hirsch $\mathrm{E}_{\text {, }}$ Jansen FE, Lagae L, Moshe SL, Peltola J, Roulet Perez E et al (2017) Operational classification of seizure types by the International League Against Epilepsy: position paper of the ILAE Commission for Classification and Terminology. Epilepsia 58(4):522-530

22. Scheffer IE, Berkovic S, Capovilla G, Connolly MB, French J, Guilhoto L, Hirsch E, Jain S, Mathern GW, Moshe SL et al (2017) ILAE classification of the epilepsies: position paper of the ILAE Commission for Classification and Terminology. Epilepsia 58(4):512-521

23. Trinka $E$, Cock $H$, Hesdorffer D, Rossetti AO, Scheffer IE, Shinnar S, Shorvon S, Lowenstein DH (2015) A definition and classification of status epilepticus-report of the ILAE Task Force on Classification of Status Epilepticus. Epilepsia 56(10):1515-1523

24. von Elm E, Altman DG, Egger M, Pocock SJ, Gotzsche PC, Vandenbroucke JP, Initiative $S$ (2008) Das Strengthening the Reporting of Observational Studies in Epidemiology (STROBE) Statement: Leitlinien für das Berichten von Beobachtungsstudien. Internist 49(6):688-693

25. Riechmann J, Strzelczyk A, Reese JP, Boor R, Stephani U, Langner C, Neubauer BA, Oberman B, Philippi H, Rochel M et al (2015) Costs of epilepsy and cost-driving factors in children, adolescents, and their caregivers in Germany. Epilepsia 56(9):1388-1397

26. Strzelczyk A, Nickolay T, Bauer S, Haag A, Knake S, Oertel WH, Reif PS, Rosenow F, Reese JP, Dodel R et al (2012) Evaluation of health-care utilization 
among adult patients with epilepsy in Germany. Epilepsy Behav 23(4):451-457

27. Riechmann J, Willems LM, Boor R, Kieslich $M$ Knake S, Langner C, Neubauer BA, Oberman B, Philippi H, Reese JP et al (2019) Quality of life and correlating factors in children, adolescents with epilepsy, and their caregivers: a cross-sectional multicenter study from Germany. Seizure 69:92-98

28. Ravens-Sieberer U, Bullinger M (1998) Assessing health-related quality of life in chronically ill children with the German KINDL:first psychometric and content analytical results. Qual Life Res 7(5):399-407

29. Toledano-Toledano F, Contreras-Valdez JA (2018) Validity and reliability of the Beck Depression Inventory II (BDI-II) in family caregivers of children with chronic diseases. PLoSONE 13(11):e206917

30. Rabin R, de Charro F (2001) EQ-5D: a measure of health status from the EuroQol Group. Ann Med 33(5):337-343

31. Strzelczyk A, Kalski M, Bast T, Wiemer-Kruel A Bettendorf U, Kay L, Kieslich M, Kluger G, Kurlemann G, Mayer T et al (2019) Burden-ofillness and cost-driving factors in Dravet syndrome patients and carers: a prospective, multicenter study from Germany. Eur J Paediatr Neurol 23(3):392-403

32. Schubert-Bast $S$, Wolff $M$, Wiemer-Kruel $A$, von Spiczak S, Trollmann R, Reif PS, Pritchard C, Polster T, Neubauer BA, Mayer T et al (2019) Seizure management and prescription patterns of anticonvulsants in Dravet syndrome: a multicenter cohort study from Germany and review of literature. Epilepsy Behav 98:88-95

33. Strzelczyk A, Schubert-Bast S, Reese JP, Rosenow F, Stephani U, Boor R (2014) Evaluation of healthcare utilization in patients with Dravet syndrome and on adjunctive treatment with stiripentol and clobazam. Epilepsy Behav 34:86-91

34. Lagae L, Brambilla I, Mingorance A, Gibson E, Battersby A (2018) Quality of life and comorbidities associated with Dravet syndrome severity: a multinational cohort survey. Dev Med Child Neurol 60(1):63-72

35. Strzelczyk A, Schubert-Bast S, Bast T, BettendorfU, Fiedler B, Hamer HM, Herting A, Kalski M, Kay L, Kieslich Metal (2019) A multicenter, matched casecontrol analysis comparing burden-of-illness in Dravet syndrome to refractory epilepsy and seizure remission in patients and caregivers in Germany. Epilepsia 60(8):1697-1710

36. Willems LM, Richter S, Watermann N, Bauer $S$ Klein KM, Reese JP, Schoffski O, Hamer HM, Knake S, Rosenow F et al (2018) Trends in resource utilization and prescription of anticonvulsants for patients with active epilepsy in Germany from 2003 to 2013-a ten-year overview. Epilepsy Behav 83:28-35

37. Lagae L, Irwin J, Gibson E, Battersby A (2019) Caregiver impact and health service use in high and low severity Dravet syndrome: a multinational cohortstudy. Seizure 65:72-79

38. Strzelczyk A, Ansorge S, Hapfelmeier J, Bonthapally V, Erder MH, Rosenow F (2017) Costs, length of stay, and mortality of super-refractory status epilepticus: a population-based study from Germany. Epilepsia 58(9):1533-1541

39. Schubert-Bast S, Zöllner JP, Ansorge S, Hapfelmeier J, Bonthapally V, Eldar-Lissai A, Rosenow F, Strzelczyk A (2019) Burden and epidemiology of status epilepticus in infants, children, and adolescents: a population-based study on German health insurance data. Epilepsia 60(5):911-920
40. Brunklaus A, Dorris L, Zuberi SM (2011) Comorbidities and predictors of health-related quality of life in Dravet syndrome. Epilepsia 52(8):1476-1482

41. Reilly C, Atkinson P, Memon A, Jones C, Dabydeen L, Das KB, Gillberg C, Neville BGR, Scott RC (2018) Symptoms of depression, anxiety, and stress in parents of young children with epilepsy: a case controlled population-based study. Epilepsy Behav 80:177-183

42. Wood LJ, Sherman EM, Hamiwka LD, Blackman MA, Wirrell EC (2008) Maternal depression: the cost of caring for a child with intractable epilepsy. Pediatr Neurol 39(6):418-422

43. Villas N, Meskis MA, Goodliffe S (2017) Dravet syndrome: characteristics, comorbidities, and caregiver concerns. Epilepsy Behav 74:81-86

44. Jensen MP, Brunklaus A, Dorris L, Zuberi SM, Knupp KG, Galer BS, Gammaitoni AR (2017) The humanistic and economic burden of Dravet syndrome on caregivers and families: implications for future research. Epilepsy Behav 70(PtA):104-109

45. Ishii $A$, Watkins JC, Chen D, Hirose $S$, Hammer MF (2017)Clinical implications of SCN1A missense and truncation variants in a large Japanese cohort with Dravet syndrome. Epilepsia 58(2):282-290

46. Lotte J, Haberlandt E, Neubauer B, Staudt M, Kluger GJ (2012) Bromide in patients with SCN1Amutations manifesting as Dravet syndrome. Neuropediatrics 43(1):17-21

47. Ernst JP, Doose H, Baier WK (1988) Bromides were effective in intractable epilepsy with generalized tonic-clonic seizures and onset in early childhood. Brain Dev 10(6):385-388

48. Kortland LM, Knake $S$, von Podewils F, Rosenow $F$ Strzelczyk A (2017) Socioeconomic outcome and quality of life in adults after status epilepticus: a multicenter, longitudinal, matched case-control analysis from Germany. Front Neurol 8:507

49. Brunklaus A, Zuberi SM (2014) Dravet syndromeFrom epileptic encephalopathy to channelopathy. Epilepsia 55(7):979-984

50. Nabbout R, Auvin S, Chiron C, Thiele E, Cross H, Scheffer IE, Schneider AL, Guerrini R, Williamson N Irwin J et al (2019) Perception of impact of Dravet syndrome on children and caregivers in multiple countries: looking beyond seizures. Dev Med Child Neurol 61(10):1229-1236 